\title{
Opportunities for Advanced Imaging Techniques in Pharmaceutical Formulation Development
}

\author{
Matthew S. Lamm ${ }^{*}$ \\ 1. Preformulation Group, Merck Research Laboratories, Merck and Co., Inc., Rahway, NJ, USA. \\ * Corresponding author: matthew.lamm@merck.com
}

The complexity of modern pharmaceutical formulation development requires increasingly sophisticated understanding of the role of composition and process on the ultimate performance of the drug product. Among the variety of analytical tools available to the pharmaceutical materials scientist, imaging plays a pivotal role in characterizing formulations precisely because of the richness of information contained within the image, especially when coupled with spectroscopic or other chemical analysis. In a formulated tablet, for example, the ability to visualize the size and shape of the components within the formulation may provide useful information as to how the individual particles have been altered as result of milling or compaction processes. However, it is the understanding of the spatial distribution of each component relative to each other that can provide deeper insight as to how effectively the materials were blended and perhaps what physical or chemical changes may be occurring at the interface of two different types of particles such as an active ingredient (drug) and excipient. In this regard, spectroscopic chemical imaging is of particular value and is continuing to gain interest within the pharmaceutical field. The Merck Preformulation group has been evaluating chemical imaging techniques beyond traditional Raman microscopy that enable faster acquisition speeds but also provide good chemical specificity such as broadband coherent anti-stokes Raman spectroscopy (CARS) microscopy[1], as well as more recently a study evaluating the ability of stimulated Raman spectroscopy (SRS) imaging to visualize the dissolution process of drug crystals embedded in a controlled release implant[2]. In addition to fast Raman imaging techniques, infrared microscopy enabled by tuneable quantum cascade lasers (QCL) can provide similar but complementary spectroscopic information with rapid acquisition times. There are multiple commercial instruments now utilizing the recent advancements in QCL technology of which have been explored for use in pharmaceutical applications.

In addition to chemical spectroscopic imaging techniques, X-ray computed tomography (XRCT) has become an increasingly important tool to understand the 3-dimensional structures of pharmaceutical formulations in a non-destructive quality control type of application where tablets are analysed for internal cracks, for example, or drug delivery devices such as inhalers are imaged to ensure appropriate dose filling. When paired with advanced image analysis and modelling/simulation, XRCT can be a very powerful tool to understand and predict the mechanism and rates of release of long acting implant formulations as both compositional and processing variables are explored[3].

For applications requiring high spatial resolution, scanning probe microscopy (SPM) can provide insight as to morphology and heterogeneity in multicomponent systems where traditional electron microscopy or chemical imaging techniques suffer from lack of contrast, spectral specificity or resolution. Within pharmaceutical formulation development, scanning probe techniques are readily applied to cases where the phase behaviour of drug, excipient, and polymers mixtures are examined especially when prepared using hot melt extrusion techniques. For these cases, alternate contact or tapping mode atomic force microscopy (AFM) with phase imaging can provide insight as to the extent of mixing and domain size of phase separated systems[4]. Furthermore, advanced scanning modes such as bimodal AFM imaging 
allowing simultaneous mapping of nanomechanical properties such as those related to elasticity, viscoelastic dissipation and tip/sample adhesion may provide additional sources of image contrast and, when calibrated/modelled appropriately, quantitative mechanical property determination. Figure 1 shows an example bimodal AFM imaging of a multicomponent modified release tablet coating where water insoluble ethyl cellulose is dispersed within a soluble hydroxypropyl methyl cellulose matrix to modulate the moisture ingress and affect tablet disintegration. For the purposes of AFM analysis, the coating solution was spin cast onto a silicon wafer. AFM imaging was operated in bimodal tapping where the cantilever was simultaneously driven at two non-harmonic resonances. The first mode is used to provide sample tracking via amplitude feedback as well as providing phase contrast, whereas the phase is locked on the second resonance to track shifts in resonant frequency. By analysing the changes in frequency and amplitude of the second cantilever vibrational mode, changes in elastic and dissipative tip/sample interactions, respectively can also be mapped.

\section{References:}

[1] CM Hartshorn et al., Analytical Chemistry 85 (2013), p. 8102

[2] AT Francis et al., Molecular Pharmaceutics 15 (2018), p. 5793

[3] R Irizarry et al., Industrial \& Engineering Chemistry Research 57 (2018), p. 15329

[4] MS Lamm et al., AAPS PharmSciTech 17 (2016), p. 89

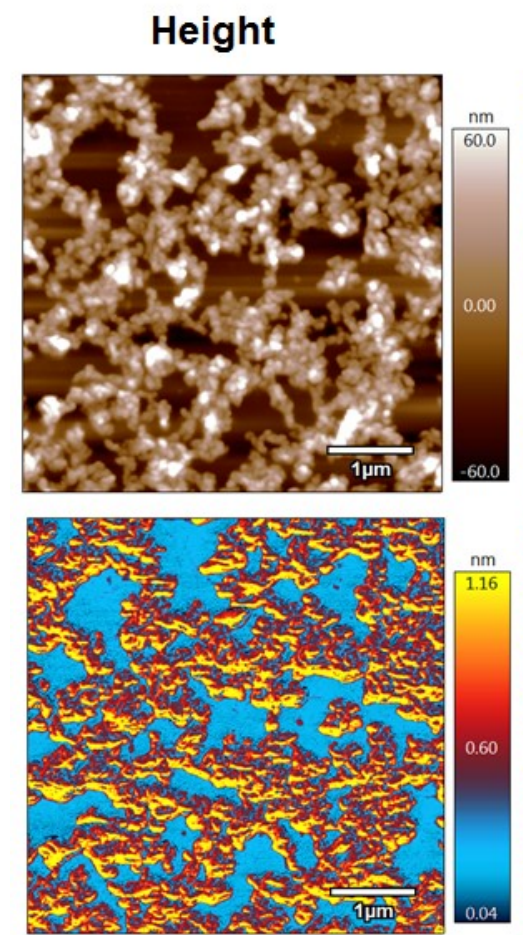

Indentation
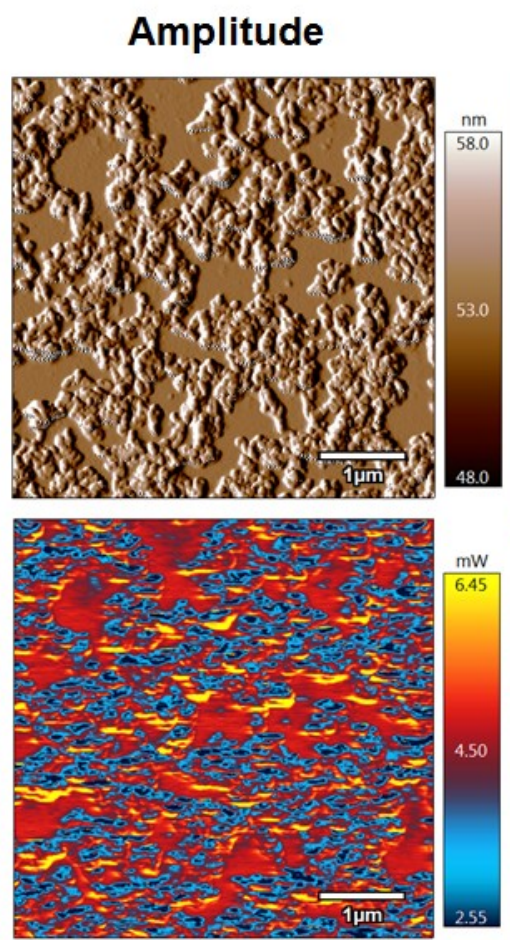

Dissipation
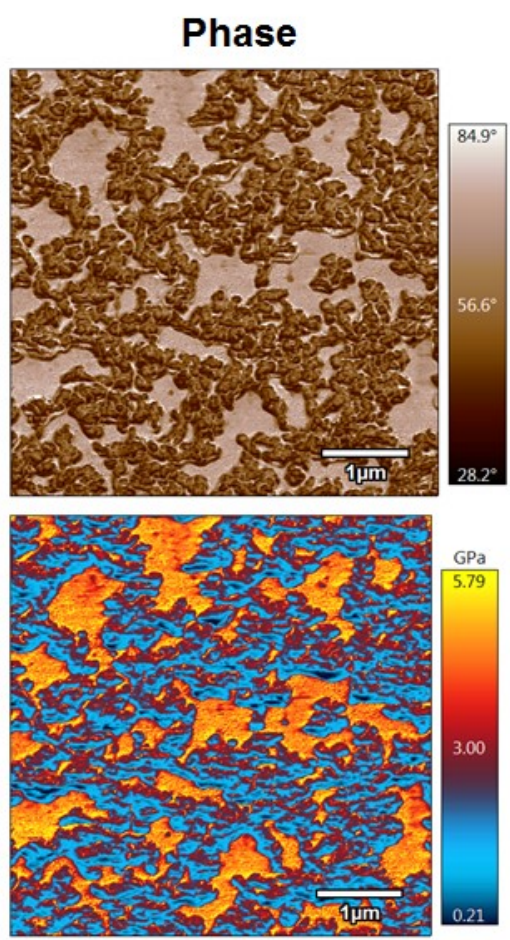

Modulus

Figure 1. Modified release tablet coating formulation imaged with bimodal AFM highlighting both topographical and nanomechanical contrast of the phase separated system. Note absolute values of modulus reported here are arbitrary as AFM tip radius was not calibrated via reference standard for this experiment. 